\title{
NONOCCURRENCE OF STABILITY SWITCHING IN SYSTEMS OF DIFFERENTIAL EQUATIONS WITH DISTRIBUTED DELAYS
}

BY

\author{
YANG KUANG
}

Arizona State University, Tempe, Arizona

\begin{abstract}
This paper deals with stability aspects of delay differential equations with general distributed delays. The objective is to show that, frequently, general distributed delays are not harder to handle than discrete delays. This is accomplished by treating two-dimensional systems of differential delay equations with distributed delays via several different approaches. All of these approaches are general, effective, and easy to apply.
\end{abstract}

1. Introduction. Frequently, models of real life systems consist of linear or nonlinear delay differential equations. Most studies on these models, as in the case of ordinary differential equations, start from the local stability analysis of some special solutions (often steady state) in order to answer the question of how delays affect the stability of these systems. For this purpose, the standard approach is to analyze the stability of the linearized equations about the special solution. If the delay differential equations are autonomous and the special solution is a constant, then the linearized equations take the form of linear autonomous delay differential equations. The stability of the trivial solution of the linearized equations depends on the location of the roots of the associated characteristic equations. General theory and applications of delay differential equations can be found in [5].

Most of the existing results are established for systems of equations with discrete delays (e.g., $[1-4,7])$. These results do not seem to have any obvious analogues for their more general distributed delay counterparts. However, a more careful second examination of their proofs may reveal some natural generalizations of some kind. This can be seen from our analysis of the following general two-dimensional system of linear differential equations with (infinite) distributed delays:

$$
\left\{\begin{array}{l}
d u(t) / d t=a_{11} u(t)+a_{12} v(t)+\int_{-\tau}^{0} u(t+\theta) d \eta_{1}(\theta)+\int_{-\tau}^{0} v(t+\theta) d \eta_{2}(\theta), \\
d v(t) / d t=a_{21} u(t)+a_{22} v(t)+\int_{-\tau}^{0} u(t+\theta) d \eta_{3}(\theta)+\int_{-\tau}^{0} v(t+\theta) d \eta_{4}(\theta),
\end{array}\right.
$$

Received August 5, 1992.

1991 Mathematics Subject Classification. Primary 34K20, 92D25.

Research supported by NSF Grant DMS-9102549. 
where $a_{i j}$ are real, $\tau \leq+\infty$, and

$$
\int_{-\tau}^{0}\left|d \eta_{i}(\theta)\right|<+\infty, \quad i=1,2,3,4,
$$

i.e., $\eta_{i}$ have finite total variations. System (1.1) has the following system as a special case:

$$
\left\{\begin{array}{l}
d u(t) / d t=a_{11} u(t)+a_{12} v(t)+\sum_{j=1}^{m} b_{1 j} u\left(t-\tau_{1 j}\right)+\sum_{j=1}^{m} b_{2} v\left(t-\tau_{2 j}\right), \\
d v(t) / d t=a_{21} u(t)+a_{22} v(t)+\sum_{j=1}^{m} c_{1 j} u\left(t-\xi_{1 j}\right)+\sum_{j=1}^{m} c_{2 j} v\left(t-\xi_{2 j}\right),
\end{array}\right.
$$

where $a_{i j}$ are real and $\tau_{1 j}, \tau_{2 j}, \xi_{1 j}, \xi_{2 j}, j=1, \ldots, m$, are nonnegative constants. System (1.2) is studied in Freedman and Gopalsamy [3], where sufficient conditions of nonoccurrence of stability switching are established. This is also our objective for system (1.1) in this paper. Our approaches in principle are similar to those of Freedman and Gopalsamy [3], which exploit the fact that stability switching is possible only when the corresponding characteristic equation has pure imaginary roots. This important observation is rigorously proved in the following lemma.

Lemma 1.1. Let $f(\lambda, \vec{\alpha})=\lambda^{n}+g(\lambda, \vec{\alpha})$, where $g(\lambda, \vec{\alpha})$ is an analytic function with respect to $\lambda$ and $\vec{\alpha}$, where $\vec{\alpha}=\left(\alpha_{1}, \ldots, \alpha_{m}\right)$ and $\operatorname{Re} \lambda>-\beta$, where $\beta$ is a positive constant. Assume that

$$
\gamma=\limsup _{\substack{\operatorname{Re} \lambda \geq 0 \\|\lambda| \rightarrow \infty}}\left|\lambda^{-n} g(\lambda, \vec{\alpha})\right|<1 .
$$

Then, as $\vec{\alpha}$ varies, the sum of the multiplicities of the roots of $f(\lambda, \vec{\alpha})=0$ in the open right half-plane can change only if a root appears on or crosses the imaginary axis.

Proof. We note that since $f(\lambda, \vec{\alpha})$ is analytic for $\operatorname{Re} \lambda>-\beta$, it can have only a finite number of zeros in any compact set of the open right half complex plane. If $f(\lambda, \vec{\alpha})$ has an infinite number of roots in the open right half-plane, then there is a sequence $\left\{\lambda_{j}\right\}$ such that $f\left(\lambda_{j}, \vec{\alpha}\right)=0,\left|\lambda_{j}\right| \rightarrow \infty$, which in turn implies that

$$
0=\frac{f\left(\lambda_{j}, \vec{\alpha}\right)}{\lambda_{j}^{n}}=1+\frac{1}{\lambda_{j}^{n}} g\left(\lambda_{j}, \vec{\alpha}\right) .
$$

Hence,

$$
\lim _{j \rightarrow \infty}\left|\lambda_{j}^{-n} g\left(\lambda_{j}, \vec{\alpha}\right)\right|=1,
$$

a contradiction to Eq. (1.3). Therefore, the total multiplicity $M(\vec{\alpha})$ of roots of $f(\lambda, \vec{\alpha})=0$ in the open right half-plane is finite. 
Let $\lambda=\lambda(\vec{\alpha})$ be any root of $f(\lambda, \vec{\alpha})=0$. If we place a small disk around $\lambda(\vec{\alpha})$, then for $\vec{\alpha}^{\prime}$ sufficient close to $\vec{\alpha}$, the total multiplicity of roots of $f\left(\lambda, \vec{\alpha}^{\prime}\right)=0$ in the disk equals the multiplicity of $\lambda(\vec{\alpha})$. Hence, from Rouchés theorem, we see that as $\vec{\alpha}$ changes, $\lambda(\vec{\alpha})$ changes accordingly, and it will not suddenly disappear or appear or change its multiplicity at a finite point in the complex plane.

Suppose that $M(\vec{\alpha})$ changes but that no roots appear on and cross the imaginary axis. This can only occur due to the appearance of a root bifurcating from infinity. That is, there exists an $\vec{\alpha}^{*}$ and a root $\lambda(\vec{\alpha})$ such that $|\lambda(\vec{\alpha})| \rightarrow \infty$ as $\vec{\alpha} \rightarrow \vec{\alpha}^{*}$, with $\operatorname{Re} \lambda(\vec{\alpha}) \geq 0$. We thus have

$$
0=\lambda^{-n}(\vec{\alpha}) f(\lambda(\vec{\alpha}), \vec{\alpha})=1+\lambda^{-n}(\vec{\alpha}) g(\lambda(\vec{\alpha}), \vec{\alpha})
$$

However, $\left|1+\lambda^{-n}(\vec{\alpha}) g(\lambda(\vec{\alpha}), \vec{\alpha})\right| \geq \frac{1}{2}(1-\gamma)>0$ for $\vec{\alpha}$ close enough to $\vec{\alpha}^{*}$. This contradicts Eq. (1.5) and proves the lemma.

2. Main results. Throughout the rest of this paper, we assume that in system (1.1), there is a positive constant $\beta$ such that

$$
\int_{-\tau}^{0} e^{-\beta \theta}\left|d \eta_{i}(\theta)\right|<+\infty, \quad i=1, \ldots, 4
$$

Hence, we must have

$$
\begin{aligned}
\bar{\eta}_{i} & \equiv \int_{-\tau}^{0}\left|d \eta_{i}(\theta)\right|<+\infty \\
\sigma_{i} & \equiv \int_{-\tau}^{0}|\theta|\left|d \eta_{i}(\theta)\right|<+\infty, \quad i=1, \ldots, 4 .
\end{aligned}
$$

Denote

$$
\begin{aligned}
& \vec{\alpha}=\left(\alpha_{1}, \alpha_{2}, \alpha_{3}, \alpha_{4}\right), \\
& g(\lambda, \vec{\alpha})=-\left(a_{11}+a_{22}\right) \lambda+a_{11} a_{22}-a_{12} a_{21} \\
&-\lambda\left[\int_{-\tau}^{0} e^{\lambda \theta} d\left(\alpha_{1} \eta_{1}(\theta)+\alpha_{4} \eta_{4}(\theta)\right)\right]+a_{11} \alpha_{4} \int_{-\tau}^{0} e^{\lambda \theta} d \eta_{4}(\theta) \\
&+a_{22} \alpha_{1} \int_{-\tau}^{0} e^{\lambda \theta} d \eta_{1}(\theta)+\alpha_{1} \alpha_{4} \int_{-\tau}^{0} e^{\lambda \theta} d \eta_{1}(\theta) \int_{-\tau}^{0} e^{\lambda \theta} d \eta_{4}(\theta) \\
&-\alpha_{2} \alpha_{3} \int_{-\tau}^{0} e^{\lambda \theta} d \eta_{2}(\theta) \int_{-\tau}^{0} e^{\lambda \theta} d \eta_{3}(\theta)-a_{12} \alpha_{3} \int_{-\tau}^{0} e^{\lambda \theta} d \eta_{3}(\theta) \\
&-a_{21} \alpha_{2} \int_{-\tau}^{0} e^{\lambda \theta} d \eta_{2}(\theta),
\end{aligned}
$$




$$
D(\lambda, \vec{\alpha})=\lambda^{2}+g(\lambda, \vec{\alpha}) .
$$

From (2.1) we see that $D(\lambda, \vec{\alpha})$ is analytic for $\operatorname{Re} \lambda \geq-\beta, \vec{\alpha} \in \mathbf{R}^{4}$; so Lemma 1.1 is applicable to $D(\lambda, \vec{\alpha})$.

The characteristic equation associated with system (1.1) is

$$
\begin{aligned}
D(\lambda,(1,1,1,1))= & \lambda^{2}-\left(a_{11}+a_{22}\right) \lambda+\left(a_{11} a_{22}-a_{12} a_{21}\right) \\
& -\lambda\left[\int_{-\tau}^{0} e^{\lambda \theta} d\left(\eta_{1}(\theta)+\eta_{4}(\theta)\right)\right] \\
& +a_{11} \int_{-\tau}^{0} e^{\lambda \theta} d \eta_{4}(\theta)+a_{22} \int_{-\tau}^{0} e^{\lambda \theta} d \eta_{1}(\theta) \\
& +\int_{-\tau}^{0} e^{\lambda \theta} d \eta_{1}(\theta) \int_{-\tau}^{0} e^{\lambda \theta} d \eta_{4}(\theta) \\
& -\int_{-\tau}^{0} e^{\lambda \theta} d \eta_{2}(\theta) \int_{-\tau}^{0} e^{\lambda \theta} d \eta_{3}(\theta) \\
& -a_{12} \int_{-\tau}^{0} d \eta_{3}(\theta)-a_{21} \int_{-\tau}^{0} d \eta_{2}(\theta)=0 .
\end{aligned}
$$

By virtue of Lemma 1.1, we know that system (1.1) may change stability only if its characteristic equation can have pure imaginary roots. In the following, we try to find conditions that imply Eq. (2.6) has no pure imaginary roots. We let $\lambda=i \omega$ in Eq. (2.6) and separate the real and imaginary parts of Eq. (2.6). We obtain

$$
\begin{aligned}
\omega^{2}- & \left(a_{11} a_{22}-a_{12} a_{21}\right) \\
= & -\omega \int_{-\tau}^{0} \sin \omega \theta d\left(\eta_{1}(\theta)+\eta_{4}(\theta)\right)+a_{11} \int_{-\tau}^{0} \cos \omega \theta d \eta_{4}(\theta) \\
& +a_{22} \int_{-\tau}^{0} \cos \omega \theta d \eta_{1}(\theta)+\int_{-\tau}^{0} \cos \omega \theta d \eta_{1}(\theta) \int_{-\tau}^{0} \cos \omega \theta d \eta_{4}(\theta) \\
& -\int_{-\tau}^{0} \sin \omega \theta d \eta_{1}(\theta) \int_{-\tau}^{0} \sin \omega \theta d \eta_{4}(\theta) \\
& -\int_{-\tau}^{0} \cos \omega \theta d \eta_{2}(\theta) \int_{-\tau}^{0} \cos \omega \theta d \eta_{3}(\theta) \\
& +\int_{-\tau}^{0} \sin \omega \theta d \eta_{2}(\theta) \int_{-\tau}^{0} \sin \omega \theta d \eta_{3}(\theta) \\
& -a_{12} \int_{-\tau}^{0} \cos \omega \theta d \eta_{3}(\theta)-a_{21} \int_{-\tau}^{0} \cos \omega \theta d \eta_{2}(\theta)
\end{aligned}
$$




$$
\begin{aligned}
\omega\left(a_{11}+a_{22}\right)= & -\omega \int_{-\tau}^{0} \cos \omega \theta d\left(\eta_{1}(\theta)+\eta_{4}(\theta)\right) \\
& +a_{11} \int_{-\tau}^{0} \sin \omega \theta d \eta_{4}(\theta)+a_{22} \int_{-\tau}^{0} \sin \omega \theta d \eta_{1}(\theta) \\
& +\int_{-\tau}^{0} \cos \omega(\theta) d \eta_{1}(\theta) \int_{-\tau}^{0} \sin \omega \theta d \eta_{4}(\theta) \\
& +\int_{-\tau}^{0} \sin \omega \theta d \eta_{1}(\theta) \int_{-\tau}^{0} \cos \omega \theta d \eta_{4}(\theta) \\
& -\int_{-\tau}^{0} \cos \omega \theta d \eta_{2}(\theta) \int_{-\tau}^{0} \sin \omega \theta d \eta_{3}(\theta) \\
& -\int_{-\tau}^{0} \sin \omega \theta d \eta_{2}(\theta) \int_{-\tau}^{0} \cos \omega \theta d \eta_{3}(\theta) \\
& -a_{12} \int_{-\tau}^{0} \sin \omega \theta d \eta_{3}(\theta)-a_{21} \int_{-\tau}^{0} \sin \omega \theta d \eta_{2}(\theta)
\end{aligned}
$$

For convenience, we denote

$$
\begin{gathered}
\mu_{1}=a_{11}+a_{22}, \quad \mu_{2}=a_{11} a_{22}-a_{12} a_{21}, \\
\beta=\bar{\eta}_{1}+\bar{\eta}_{4}, \quad \gamma=\left|a_{11}\right| \bar{\eta}_{4}+\left|a_{22}\right| \bar{\eta}_{1}+\left|a_{12}\right| \bar{\eta}_{3}+\left|a_{21}\right| \bar{\eta}_{2}, \\
\delta=\bar{\eta}_{1} \bar{\eta}_{4}+\bar{\eta}_{2} \bar{\eta}_{3} .
\end{gathered}
$$

If we denote by $f(\omega)$ the sum of the squares of the right sides of Eqs. (2.7) and (2.8), then after some algebraic simplifications, we have

$$
f(\omega) \leq \beta^{2} \omega^{2}+4 \beta(\gamma+\delta) \omega+4 \gamma \delta+\gamma^{2}+\delta^{2}
$$

where we make use of the observation

$$
\begin{aligned}
\left(\int_{-\tau}^{0} \cos \omega \theta d \eta(\theta)\right)^{2} & \leq \int_{-\tau}^{0} \cos ^{2} \omega \theta d \eta(\theta) \int_{-\tau}^{0} d \eta(\theta) \\
\left(\int_{-\tau}^{0} \sin \omega \theta d \eta(\theta)\right)^{2} & \leq \int_{-\tau}^{0} \sin ^{2} \omega \theta d \eta(\theta) \int_{-\tau}^{0} d \eta(\theta) \\
2 a b & \leq a^{2}+b^{2}
\end{aligned}
$$

Hence, we have

$$
\omega^{4}+\left(\mu_{1}^{2}-2 \mu_{2}\right) \omega^{2}+\mu_{2}^{2} \leq \beta^{2} \omega^{2}+2 \beta(\gamma+\delta) \omega+(\gamma+\delta)^{2} .
$$

A sufficient condition for there to be no stability switches is that Eq. (2.10) not be satisfied for any real $\omega$. This is equivalent to

$$
g(\omega) \equiv \omega^{4}+\left(\mu_{1}^{2}-2 \mu_{2}-\beta^{2}\right) \omega^{2}-2 \beta(\gamma+\delta) \omega-(\gamma+\delta)^{2}+\mu_{2}^{2}>0,
$$

for all real $\omega$. Note that Eq. (2.11) can be written as

$$
\omega^{4}+\left(\mu_{1}^{2}-2 \mu_{2}-\beta^{2}\right)\left[\omega-\frac{\beta(\gamma+\delta)}{\mu_{1}^{2}-2 \mu_{2}-\beta^{2}}\right]^{2}+\mu_{2}-(\gamma+\delta)^{2}-\frac{\beta^{2}(\gamma+\delta)^{2}}{\mu_{1}^{2}-2 \mu_{2}-\beta^{2}}>0,
$$

for all real $\omega$. 
TheOREM 2.1. The trivial solution of system (1.1) has the same stability for all $\eta_{i}(\theta)$, where

$$
\bar{\eta}_{i}=\int_{-\tau}^{0}\left|d \eta_{i}(\theta)\right|, \quad i=1,2,3,4,
$$

if

(i) $\mu_{1}^{2}-2 \mu_{2}-\beta^{2}>0$,

(ii) $\left(\mu_{2}^{2}-(\gamma+\delta)^{2}\right)\left(\mu_{1}^{2}-2 \mu_{2}-\beta^{2}\right)>\beta^{2}(\gamma+\delta)^{2}$.

Proof. Observe that if we replace $\eta_{i}(\theta)$ by $\alpha_{i} \eta_{i}(\theta), 0 \leq \alpha_{i} \leq 1, i=1,2,3,4$, then $\beta, \gamma$, and $\delta$ are replaced by smaller or equal numbers and (i) and (ii) remain true. In other words, $D(\lambda, \vec{\alpha})$ has no pure imaginary roots for all $\alpha_{i} \in[0,1]$, $i=1,2,3,4$. By Lemma 1.1 , we see that $D(\lambda,(1,1,1,1))$ has the same number of roots with positive real parts as that of $D(\lambda,(0,0,0,0))$. This is equivalent to saying that the trivial solution of (1.1) has the same stability as that of

$$
\begin{aligned}
& \dot{u}(t)=a_{11} u(t)+a_{12} v(t), \\
& \dot{v}(t)=a_{21} u(t)+a_{22} v(t),
\end{aligned}
$$

provided that (i) and (ii) hold. This proves the theorem.

The above result generalizes the main theorem of Freedman and Gopalsamy [3].

Observe that the absolute value of the right-hand side of Eq. (2.8) is less than or equal to

$$
|\omega|\left(\bar{\eta}_{1}+\bar{\eta}_{4}+\left|a_{11}\right| \sigma_{4}+\left|a_{22}\right| \sigma_{1}+\bar{\eta}_{1} \sigma_{4}+\bar{\eta}_{4} \sigma_{1}+\bar{\eta}_{2} \sigma_{3}+\bar{\eta}_{3} \sigma_{2}+\left|a_{12}\right| \sigma_{3}+\left|a_{21}\right| \sigma_{2}\right) .
$$

We thus have

THEOREM 2.2. The trivial solution of system (1.1) has the same stability for all $\eta_{i}(\theta)$ satisfying Eqs. (2.2) if

$$
\begin{aligned}
\left|a_{11}+a_{22}\right|> & \bar{\eta}_{1}+\bar{\eta}_{4}+\left|a_{11}\right| \sigma_{4}+\left|a_{22}\right| \sigma_{1}+\bar{\eta}_{1} \sigma_{4}+\bar{\eta}_{4} \sigma_{1} \\
& +\bar{\eta}_{2} \sigma_{3}+\bar{\eta}_{3} \sigma_{2}+\left|a_{12}\right| \sigma_{3}+\left|a_{21}\right| \sigma_{2} .
\end{aligned}
$$

Note that $\sigma_{i} \leq \tau \bar{\eta}_{i}$. We have

Corollary 2.3. The trivial solution of system (1.1) has the same stability for all $\eta_{i}(\theta)$ such that $\bar{\eta}_{i}=\int_{-\tau}^{0}\left|d \eta_{i}(\theta)\right|$ if

$$
0 \leq \tau<\frac{\left|a_{11}+a_{22}\right|-\bar{\eta}_{1}-\bar{\eta}_{4}}{2 \bar{\eta}_{1} \bar{\eta}_{4}+2 \bar{\eta}_{2} \bar{\eta}_{3}+\left|a_{11}\right| \bar{\eta}_{4}+\left|a_{22}\right| \bar{\eta}_{1}+\left|a_{12}\right| \bar{\eta}_{3}+\left|a_{21}\right| \bar{\eta}_{2}} .
$$


Assume now that $\bar{\eta}_{1}=\bar{\eta}_{4}=0$. Then from Eq. (2.7) we have

$$
\begin{aligned}
\omega^{2}- & \left(a_{11} a_{22}-a_{12} a_{21}\right) \\
= & -\int_{-\tau}^{0} \cos \omega \theta d \eta_{2}(\theta) \int_{-\tau}^{0} \cos \omega \theta d \eta_{3}(\theta) \\
& +\int_{-\tau}^{0} \sin \omega \theta d \eta_{2}(\theta) \int_{-\tau}^{0} \sin \omega \theta d \eta_{3}(\theta) \\
& -a_{12} \int_{-\tau}^{0} \cos \omega \theta d \eta_{3}(\theta)-a_{21} \int_{-\tau}^{0} \cos \omega \theta d \eta_{2}(\theta) \\
\geq & -\frac{1}{2} \sum_{i=2}^{3}\left[\left(\int_{-\tau}^{0} \cos \omega \theta d \eta_{i}(\theta)\right)^{2}+\left(\int_{-\tau}^{0} \sin \omega \theta d \eta_{i}(\theta)\right)\right]^{2} \\
& -\left|a_{12}\right| \bar{\eta}_{3}-\left|a_{21}\right| \bar{\eta}_{2} \\
\geq & -\frac{1}{2}\left(\bar{\eta}_{2}^{2}+\bar{\eta}_{3}^{2}\right)-\left|a_{12}\right| \bar{\eta}_{3}-\left|a_{21}\right| \bar{\eta}_{2},
\end{aligned}
$$

and similarly,

$$
\omega^{2}-\left(a_{11} a_{22}-a_{12} a_{21}\right) \geq-2 \bar{\eta}_{2} \bar{\eta}_{3}-\left|a_{12}\right| \bar{\eta}_{3}-\left|a_{21}\right| \bar{\eta}_{2} .
$$

Thus,

$$
\omega^{2}-\left(a_{11} a_{22}-a_{12} a_{21}\right) \geq \max \left\{-2 \bar{\eta}_{2} \bar{\eta}_{3},-\frac{1}{2}\left(\bar{\eta}_{2}^{2}+\bar{\eta}_{3}^{2}\right)\right\}-\left|a_{12}\right| \bar{\eta}_{3}-\left|a_{21}\right| \bar{\eta}_{2}
$$

and Eq. (2.8) reduces to

$$
\begin{aligned}
\omega\left(a_{11}+a_{22}\right)= & -\int_{-\tau}^{0} \cos \omega \theta d \eta_{2}(\theta) \int_{-\tau}^{0} \sin \omega \theta d \eta_{3}(\theta) \\
& -\int_{-\tau}^{0} \sin \omega \theta d \eta_{2}(\theta) \int_{-\tau}^{0} \cos \omega \theta d \eta_{3}(\theta) \\
& -a_{12} \int_{-\tau}^{0} \sin \omega \theta d \eta_{3}(\theta)-a_{21} \int_{-\tau}^{0} \sin \omega \theta d \eta_{2}(\theta) .
\end{aligned}
$$

From Eq. (2.17), we have

$$
\begin{aligned}
\left|\omega\left(a_{11}+a_{22}\right)\right| \leq & \left|\int_{-\tau}^{0} \cos \omega \theta d \eta_{2}(\theta) \int_{-\tau}^{0} \sin \omega \theta d \eta_{3}(\theta)\right| \\
& +\left|\int_{-\tau}^{0} \sin \omega \theta d \eta_{2}(\theta) \int_{-\tau}^{0} \cos \omega \theta d \eta_{3}(\theta)\right| \\
& +\left|a_{12} \int_{-\tau}^{0} \sin \omega \theta d \eta_{3}(\theta)\right|+\left|a_{21} \int_{-\tau}^{0} \sin \omega \theta d \eta_{2}(\theta)\right| \\
\leq & \frac{1}{2}\left(\bar{\eta}_{2}^{2}+\bar{\eta}_{3}^{2}\right)+\left|a_{12}\right| \bar{\eta}_{3}+\left|a_{21}\right| \bar{\eta}_{2} .
\end{aligned}
$$

Similarly, we have

$$
\left|\omega\left(a_{11}+a_{22}\right)\right| \leq 2 \bar{\eta}_{2} \bar{\eta}_{3}+\left|a_{12}\right| \bar{\eta}_{3}+\left|a_{21}\right| \bar{\eta}_{2} .
$$


Also, we have

$$
\begin{aligned}
\left|\omega\left(a_{11}+a_{22}\right)\right| \leq & \bar{\eta}_{2} \omega \int_{-\tau}^{0}\left|\theta d \eta_{3}(\theta)\right|+\bar{\eta}_{3} \omega \int_{-\tau}^{0}\left|\theta d \eta_{2}(\theta)\right| \\
& +\left|a_{12}\right| \omega \int_{-\tau}^{0}\left|\theta d \eta_{3}(\theta)\right|+\left|a_{21}\right| \omega \int_{-\tau}^{0}\left|\theta d \eta_{2}(\theta)\right| \\
= & \omega\left[\bar{\eta}_{2} \sigma_{3}+\bar{\eta}_{3} \sigma_{2}+\left|a_{12}\right| \sigma_{3}+\left|a_{21}\right| \sigma_{2}\right]
\end{aligned}
$$

We can summarize the above arguments as:

THEOREM 2.4. In system (1.1), assume that $\bar{\eta}_{1}=\bar{\eta}_{4}=0$ and $a_{11} a_{22}-a_{12} a_{21}>\Delta$, where

$$
\Delta=\min \left\{2 \bar{\eta}_{2} \bar{\eta}_{3}, \frac{1}{2}\left(\bar{\eta}_{2}^{2}+\bar{\eta}_{3}^{2}\right)\right\}+\left|a_{12}\right| \bar{\eta}_{3}+\left|a_{21}\right| \bar{\eta}_{2} \text {. }
$$

If

(i) $\left(a_{11}+a_{22}\right)^{2}\left(a_{11} a_{22}-a_{12} a_{21}-\Delta\right)>\Delta^{2}$ or

(ii) $\left|a_{11}+a_{22}\right|>\bar{\eta}_{2} \sigma_{3}+\bar{\eta}_{3} \sigma_{2}+\left|a_{12}\right| \sigma_{3}+\left|a_{21}\right| \sigma_{2}$,

then system (1.1) does not undergo stability switching; that is, it has the same stability as when $\bar{\eta}_{2}=\bar{\eta}_{3}=0$.

Proof. (i) is obvious from Eqs. (2.16), (2.18), and (2.19); (ii) is clear from Eq. (2.20).

An alternative approach in this case $\left(\bar{\eta}_{1}=\bar{\eta}_{4}=0\right)$ is to make use of Eq. (2.11). Now we have $\beta=0, \gamma=\left|a_{12}\right| \bar{\eta}_{3}+\left|a_{21}\right| \bar{\eta}_{2}$, and $\delta=\bar{\eta}_{2} \bar{\eta}_{3}$. Hence

$$
\begin{aligned}
g(\omega) & =\omega^{4}+\left(\mu_{1}^{2}-2 \mu_{2}\right) \omega^{2}+\mu_{2}^{2}-(\gamma+\delta)^{2} \\
& =\left[\omega^{2}+\frac{1}{2}\left(\mu_{1}^{2}-2 \mu_{2}\right)\right]^{2}+\mu_{2}^{2}-(\gamma+\delta)^{2}-\frac{1}{4}\left(\mu_{1}^{2}-2 \mu_{2}\right)^{2} .
\end{aligned}
$$

Therefore, we obtain

THEOREM 2.5. In system (1.1), assume that $\bar{\eta}_{1}=\bar{\eta}_{4}=0$ and

(i) $\mu_{2}^{2}-(\gamma+\delta)^{2}-\frac{1}{4}\left(\mu_{1}^{2}-2 \mu_{2}\right)^{2}>0$ or

(ii) $\mu_{1}^{2} \geq 2 \mu_{2}$ and $\mu_{2}^{2}>(\gamma+\delta)^{2}$.

Then system (1.1) does not undergo stability switching.

Proof. In both cases, we have $g(\omega)>0$ for $\omega \geq 0$.

REMARK. Theoretically speaking, the condition $\bar{\eta}_{1}=\bar{\eta}_{4}=0$ is not essential in the considerations presented prior to Theorem 2.4. Without $\bar{\eta}_{1}=\bar{\eta}_{4}=0$, Eq. (2.16) will become an inequality of the form $\omega^{2}+A \omega+B \geq 0$, where $A$ and $B$ are functions of $a_{i j}$ and $\eta_{i}$. In this case, we can still obtain a lower bound for $\omega$, which may be more complicated.

3. Applications. Results obtained in the previous section may appear to be complicated. Below we present two simple examples that illustrate their applications. Consider first

$$
\ddot{x}(t)+a_{1} \dot{x}(t)+a_{0} x(t)=\int_{-\tau}^{0} x(t+\theta) d \eta(\theta),
$$


where $\bar{\eta}=\int_{-\tau}^{0}|d \eta(\theta)|<\infty$ and there is a $v>0$ such that $\int_{-\tau}^{0} e^{-v \theta}|d \eta(\theta)|<+\infty$. Denote $\sigma=\int_{-\tau}^{0}|\theta d \eta(\theta)|$. The following theorem generalizes Theorems 3.22 and 3.23 in [7], which deal only with discrete delay equations.

THEOREM 3.1. The trivial solution of Eq. (3.1) is uniformly asymptotically stable if $\alpha_{0}>\bar{\eta}$ and

(i) $a_{1}>\bar{\eta}\left(a_{0}-\bar{\eta}\right)^{-1 / 2}$ or

(ii) $a_{1}>\sigma$.

Proof. Denote $x(t)=u(t), \dot{x}(t)=v(t)$. Equation (3.1) is equivalent to

$$
\left\{\begin{array}{l}
\dot{u}(t)=v(t) \\
\dot{v}(t)=-a_{0} u(t)-a_{1} v(t)+\int_{-\tau}^{0} u(t+\theta) d \eta(\theta) .
\end{array}\right.
$$

In the following, we apply Theorem 2.4 to system (3.2). We have

$$
\begin{gathered}
a_{11}=0, \quad a_{12}=1, \quad a_{21}=-a_{0}, \quad a_{22}=-a_{1}, \\
\bar{\eta}_{1}=\bar{\eta}_{2}=\bar{\eta}_{4}=0, \quad \bar{\eta}_{3}=\bar{\eta} .
\end{gathered}
$$

Hence, $\Delta=\bar{\eta}, \sigma_{3}=\sigma, a_{11}+a_{22}=-a_{1}$, and $a_{11} a_{22}-a_{12} a_{21}=a_{0} \cdot a_{0}>\bar{\eta}$ implies that $a_{11} a_{22}-a_{12} a_{21}>\Delta$. Condition (i) of Theorem 2.4 reduces to

$$
a_{1}^{2}\left(a_{0}-\bar{\eta}\right)>\bar{\eta}^{2}
$$

which coincides with (i) in the theorem. Condition (ii) of Theorem 2.4 becomes $\left|a_{1}\right|>\sigma$, which is satisfied by (ii) of the theorem.

When $\bar{\eta}=\sigma=0$, both (i) and (ii) imply that $a_{1}>0$, and, clearly, the trivial solution of

$$
\ddot{x}(t)+a_{1} \dot{x}(t)+a_{0} x(t)=0
$$

is uniformly asymptotically stable. The conclusion thus follows from Theorem 2.4.

Our next example illustrates that the principle idea utilized in the previous section can easily be applied to first-order equations. Consider

$$
\dot{x}(t)+a x(t)=b \int_{-\infty}^{0} x(t+\theta) d \eta(\theta),
$$

where $a \geq 0, b>0$ are constants and $\eta(\theta)$ is a nonconstant and nonincreasing function with bounded variation. The following theorem generalizes Theorem 3.28 in [7] and Theorem 1 in [6].

THEOREM 3.2. The trivial solution of Eq. (3.3) is uniformly asymptotically stable if

$$
a \geq 0, \quad b \int_{-\infty}^{0} \theta d \eta(\theta)<1,
$$

and there exists a constant $v>0$ such that

$$
\int_{-\infty}^{0} e^{-v \theta}|d \eta(\theta)|<+\infty
$$


Proof. Assume first that $a>0$. Clearly, when $b=0$, Eq. (3.3) is uniformly asymptotically stable. The characteristic equation of Eq. (3.3) is

$$
D(\lambda)=\lambda+a-b \int_{-\infty}^{0} e^{\lambda \theta} d \eta(\theta)=0 .
$$

Clearly, $D(\lambda)$ is analytic. $D(i \omega)=0$ implies that

$$
\begin{aligned}
& a-b \int_{-\infty}^{0} \cos (\omega \theta) d \eta(\theta)=0, \\
& \omega-b \int_{-\infty}^{0} \sin (\omega \theta) d \eta(\theta)=0 .
\end{aligned}
$$

However, for $\omega>0$, we have from (3.4)

$$
\omega-b \int_{-\infty}^{0} \sin (\omega \theta) d \eta(\theta)>\omega\left(1-b \int_{-\infty}^{0} \theta d \eta(\theta)\right)>0 .
$$

Hence, for $a>0,(3.4)$ is uniformly asymptotically stable.

By continuity, when $a=0$, Eq. (3.5) has no roots with positive real parts. The same argument as above also shows that it cannot have pure imaginary roots. If $\lambda_{j}=\rho_{j}+i \omega_{j}, j=1,2, \ldots$, are roots of Eq. (3.5) such that $\rho_{j} \rightarrow 0$ as $j \rightarrow \infty$, then

$$
\begin{array}{r}
\rho_{j}+a-b \int_{-\infty}^{0} e^{\rho_{j} \theta} \cos \left(\omega_{j} \theta\right) d \eta(\theta)=0, \\
\omega_{j}-b \int_{-\infty}^{0} e^{\rho_{j} \theta} \sin \left(\omega_{j} \theta\right) d \eta(\theta)=0 .
\end{array}
$$

Since $\int_{-\infty}^{0} e^{-v \theta}|d \eta(\theta)|<+\infty$, there is a $T>0, v / 2>\varepsilon>0$ such that $\left|\theta e^{-v \theta / 2}\right|<$ $e^{-v \theta}$ for $\theta<-T$ and

$$
b\left(\int_{-T}^{0} e^{-\varepsilon \theta} \theta d \eta(\theta)+\int_{-\infty}^{-T} e^{-v \theta / 2} \theta d \eta(\theta)\right)<1 .
$$

Hence, for $\rho_{j}>-\varepsilon$, Eq. (3.9) fails to hold. This proves the theorem.

\section{REFERENCES}

[1] K. L. Cooke and Z. Grossman, Discrete delay, distributed delay and stability switches, J. Math. Anal. Appl. 86, 592-627 (1982)

[2] K. L. Cooke and P. van den Driessche, On zeros of some transcendental equations, Funkcial. Ekvac. 29, 77-90 (1986)

[3] H. I. Freedman and K. Gopalsamy, Nonoccurrence of stability switching in systems with discrete delays, Canad. Math. Bull. 31, 52-58 (1988)

[4] H. I. Freedman and Y. Kuang, Stability switches in linear scalar neutral delay equations, Funkcial. Ekvac. 34, 187-209 (1991)

[5] J. K. Hale, Theory of functional differential equations, Springer-Verlag, New York, 1977

[6] $\mathrm{H}$. W. Stech, The effect of time lags on the stability of the equilibrium state of a population growth equation, J. Math. Biol. 5, 115-120 (1978)

[7] G. Stépán, Retarded dynamical systems: Stability and characteristic functions, Longman Scientific and Technical, Essex, 1989 